\title{
A density-based topology optimization methodology for thermoelectric energy conversion problems
}

\author{
Lundgaard, Christian; Sigmund, Ole
}

Published in:

Structural and Multidisciplinary Optimization

Link to article, DOI:

$10.1007 / \mathrm{s} 00158-018-1919-1$

Publication date:

2018

Document Version

Peer reviewed version

Link back to DTU Orbit

Citation (APA):

Lundgaard, C., \& Sigmund, O. (2018). A density-based topology optimization methodology for thermoelectric energy conversion problems. Structural and Multidisciplinary Optimization, 57(4), 1427-1442.

https://doi.org/10.1007/s00158-018-1919-1

\section{General rights}

Copyright and moral rights for the publications made accessible in the public portal are retained by the authors and/or other copyright owners and it is a condition of accessing publications that users recognise and abide by the legal requirements associated with these rights.

- Users may download and print one copy of any publication from the public portal for the purpose of private study or research.

- You may not further distribute the material or use it for any profit-making activity or commercial gain

- You may freely distribute the URL identifying the publication in the public portal

If you believe that this document breaches copyright please contact us providing details, and we will remove access to the work immediately and investigate your claim 


\title{
A density-based topology optimization methodology for thermoelectric energy conversion problems
}

\author{
Christian Lundgaard $^{\mathrm{a}} \cdot$ Ole Sigmund $^{\mathrm{a}}$
}

Received: date / Accepted: date

\begin{abstract}
A density-based topology optimization approach for thermoelectric (TE) energy conversion problems is proposed. The approach concerns the optimization of thermoelectric generators (TEGs) and thermoelectric coolers (TECs). The framework supports convective heat transfer boundary conditions, temperature dependent material parameters and relevant objective functions. Comprehensive implementation details of the methodology are provided, and seven different design problems are solved and discussed to demonstrate that the approach is well-suited for optimizing TEGs and TECs. The study reveals new insight in TE energy conversion, and the study provides guidance for future research, which pursuits the development of high performing and industrially profitable TEGs and TECs.
\end{abstract}

\section{Introduction}

This paper presents a density-based topology optimization approach (Bendsøe and Sigmund, 2003, Bendsøe and Kikuchi, 1988, Sigmund and Maute, 2013) for nonlinear strongly coupled thermoelectric (TE) energy conversion problems. Thermoelectricity is a multi-physical problem which concerns the interaction and coupling between electric and thermal energy in semi conducting materials (Goldsmid, 2009, Rowe, 2005). TE energy conversion is an interesting and important engineering field due to the globally increasing demand on non-polluting and renewable energy resources. The increasing demand is predicted by many researchers to be partly covered by TE energy conversion in e.g. large scale commercial

\footnotetext{
a Department of Mechanical Engineering

Techinical University of Denmark, Richard Petersens Plads Building 322, DK-2800 Kgs. Lyngby, Denmark

E-mail: chrlund@mek.dtu.dk
}

waste heat recovery and cooling applications (Champier, 2017). Improvements in efficiencies of thermoelectric generators (TEGs) and thermoelectric coolers (TECs) are required to make TE energy conversion economically profitable and competitive with conventional waste heat recovery and cooling technologies (Vining, 2009). As TE energy conversion is predicted to have large-scale application perspectives, a topology optimization approach as presented in this study, may be used to reach important performance improvements. The topology optimization approach provides a road to systematically optimize an arbitrary TE energy conversion problem with respect to realistic boundary conditions, arbitrary dimensions and length-scales, realistic non-linear material parameters and relevant objective functions.

Thermoelectric energy conversion is described by two separately identified effects: The Seebeck effect and the Peltier effect. The Seebeck effect concerns the conversion of thermal energy into electric energy and the Peltier effect concerns the conversion of electric energy into thermal energy (Goldsmid, 2009, Rowe, 2005).

A TE device (thermoelectric generator (TEG) or thermoelectric cooler (TEC) is sketched in Fig. 1a, and a TE module is sketched in Fig. 1b. With reference to Fig. 1a, a TE device consists of three major parts: legs consisting of TE active materials which drive the TE energy conversion (components colored with blue and

); electric conductors which connect the TE legs electrically (components colored with gray) and substrates which constitute the interface between the heat source and cooling sink (components colored with dark gray). With reference to Fig. 1b, a TE module consists of two dissimilar types of semiconductors: $n$-types legs which are charged negatively and $p$-types legs which are charged positively. If $p$ and $n$-type legs are subject to a 
temperature gradient in the same direction, the legs will generate electric potential gradients in opposite directions. If a $p$ and $n$-type legs are subjected to an electric potential difference in different directions, the legs will be subject to heat fluxes in the same direction. The TE device in Fig. 1 is electrically in series and thermally in parallel. This configuration is utilized to build up a working electric potential over the device for TEGs and direct the thermal energy transfer from one surface to the other for TECs.

The efficiency of TE devices depends on the type of TE active materials utilized. The efficiency of a TE material is positively correlated with the Seebeck coefficient, $\alpha$, positively correlated with the electric conductivity, $\sigma$, and negatively correlated with the thermal conductivity, $\kappa$. A high $\alpha$ facilitates a large amount of Seebeck and Peltier work for a given temperature gradient or electric potential gradient. A high $\sigma$ facilitates a low electric energy loss due to Joule heating. A low $\kappa$ facilitates a large temperature gradient between the heat source and the cooling sink. The TE figure-of-merit, $Z T=\alpha^{2} \sigma / \kappa T$, where $T$ is the temperature $[\mathrm{K}]$, is prone to much scientific attention in the thermoelectric society (Tritt and Subramanian, 2006, Yamashita et al., 2003, Yang et al., 2012), as the magnitude of $Z T$ of a TE material is positively correlated with the ability and efficiency of the material to carry out thermoelectric energy conversion. However, we believe that the end goal device application is a better performance measure than $Z T$, for which reason we in this study focus on objectives such as electric power output, $f_{P}$, electric conversion efficiency, $f_{\eta}$, temperature, $f_{T}$, thermal heat flux, $f_{Q}$ and coefficient of performance $f_{\mu}$.

The topology optimization approach presented in this study takes basis in the idea of distributing two different TE active materials in a two dimensional design space in order to optimize for a performance measure such as $f_{P}, f_{\eta}, f_{T}, f_{Q}$ or $f_{\mu}$.

Topology optimization for TE energy conversion problems are related to the topology optimization of piezoelectric actuators with respect to the governing physics and the boundary conditions. Piezoelectric actuators have been investigated in the e.g. Sigmund (1998).

A topology optimization approach for TEGs has been proposed previously in the work by Takezawa and Kitamura (2012). Takezawa and Kitamura proposed a single material COMSOL-based topology optimization framework which supported $f_{P}$ and $f_{\eta}$ objectives and temperature dependent materials. The design solutions were primarily governed by an active volume constraint, a solid-void material phase formulation, an L-shaped design domain and fixed temperature boundary conditions between the boundaries of heat source and cooling sink. The methodology proposed in this study facilitates a completely different design problem, as two TE material phases are distributed in the design space. The configuration with two design phases and no void in the design domain complies with realistic configurations of TEGs and TECs.

Heghmanns and Beitelschmidt (2015) presented a genetic approach to optimize the electric power output and the thermo-mechanical-stress for TEGs. Heghmanns and Beitelschmidt parameterized the height of the insulators; and the number, the width and the height of the TE legs. Such approaches are not applicable to topology optimization as discussed in Sigmund (2011).

Analytically founded approaches for TEGs have been presented in the works of Sakai et al. (2014), Ursell and Snyder (2002), Yang et al. (2012, 2013). The approaches took basis in two materials phase optimization for the non-dimensional figure-of-merit, the electric power output and the conversion efficiency for segmented and off-diagonal problems. All these approaches were limited to fixed temperature boundary conditions, simple topological design solutions and constant material parameters.

The topology optimization approach presented in this study supports TEGs and TECs relevant application objectives, physically realistic convective heat transfer boundary conditions, temperature dependent material parameters, and full control of length-scales and device dimensions. The numerical framework provides a road to systematic optimization of TEGs and TECs in industrially relevant settings. The study demonstrates that optimal geometric designs of TEGs and TECs depend on many factors, such as material parameters, boundary conditions, length-scales, model dimensions and objective functions. Furthermore, it is possible to achieve design performance of two materials which exceed the performance of the individual materials. The primary aim of the study is to communicate the methodology and test the approach on academic problems, however, the approach can straightforwardly be applied to other and/or industrially relevant designs problems.

The paper is organized as follows. The physical model is introduced in Sec. 2, the finite element formulation is introduced in Sec. 3, the topology optimization framework is introduced in Sec. 4, the implementation details are covered in Sec. 5, numerical examples are presented in Sec. 6, and finally, Sec. 7 contains discussions and conclusions. 


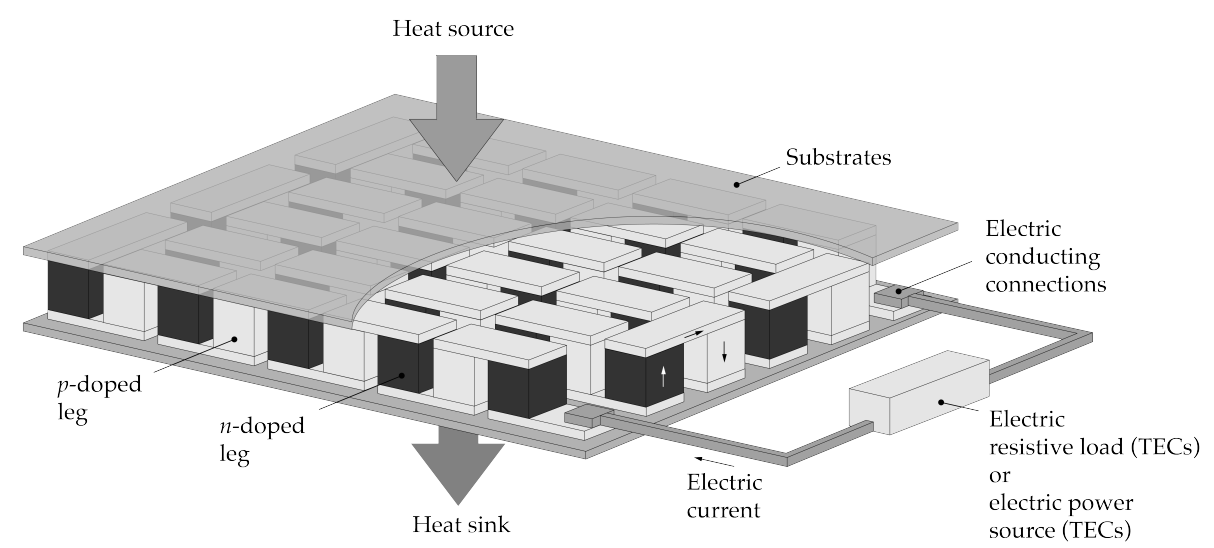

(a) TE device

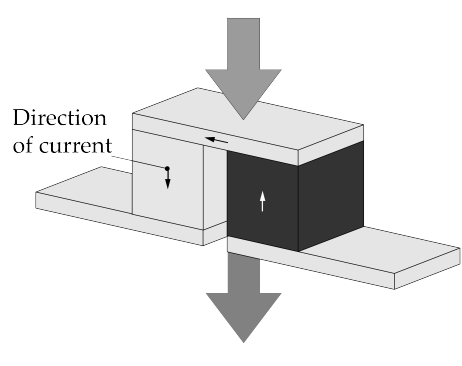

(b) TE module

Fig. 1: A schematic of a TE device and TE module

\section{Physical model}

The continuity of thermal energy and electric charge is in an arbitrary domain, $\Omega$, given by:

$$
\begin{array}{lll}
\nabla_{x} \cdot \mathbf{Q}=\dot{q} & \text { in } & \Omega \\
\nabla_{x} \cdot \mathbf{J}=0 & \text { in } \quad
\end{array}
$$

where $\mathbf{Q}$ is the heat flow density $\left[\mathrm{W} / \mathrm{m}^{2}\right] ; \nabla_{x}$ denotes the spatial derivative with respect to Cartesian directions $x$ and $y ; \dot{q}=\mathbf{J} \cdot \mathbf{E}$ is the Joule heating term $\left[\mathrm{W} / \mathrm{m}^{3}\right]$; $\mathbf{E}=-\nabla V$ is the electric field $[\mathrm{V} / \mathrm{m}]$ and $\mathbf{J}$ is the electric current density $\left[\mathrm{A} / \mathrm{m}^{2}\right]$. In thermoelectric analysis, the thermal and electric energies are coupled by the constitutive equations (Rowe, 2005):

$\mathbf{Q}=T \boldsymbol{\alpha} \cdot \mathbf{J}-\kappa \cdot \nabla_{x} T$

$\mathbf{J}=\boldsymbol{\sigma} \cdot\left(\mathbf{E}-\alpha \cdot \nabla_{x} T\right)$

where $T$ is the temperature $[\mathrm{K}] ; V$ is the electric potential; $\alpha$ is the Seebeck coefficient $[\mathrm{V} / \mathrm{K}] ; \kappa$ is the thermal conductivity $[\mathrm{W} / \mathrm{m} \cdot \mathrm{K}]$ and $\sigma$ is the electrical conductivity $[\mathrm{S} / \mathrm{m}]$. The material parameters are temperature dependent for which reason $\alpha=\alpha(T), \sigma=\sigma(T), \kappa=\kappa(T)$. The boundary conditions for Eqs. (1)-(4) are:

$$
\begin{aligned}
\text { Fixed electric potential: } & V=c_{1} \\
\text { Fixed temperature: } & T=c_{2} \\
\text { Thermal insulation: } & \mathbf{n} \cdot \mathbf{Q}=0 \\
\text { Electric insulation: } & \mathbf{n} \cdot \mathbf{J}=0 \\
\text { Electric current in outer load: } & \mathbf{n} \cdot \mathbf{J}=c_{3} \\
\text { Electrodes on boundary: } & \mathbf{r} \cdot \mathbf{J}=c_{4} \\
\text { Convective heat transfer: } & \mathbf{n} \cdot \mathbf{Q}=c_{5}
\end{aligned}
$$

where $\mathbf{n}$ is a vector normal to the boundary, where the boundary condition is imposed; $\mathbf{r}$ is a vector perpendicular to the boundary where the boundary condition is imposed; and $c_{1}, c_{2}, c_{3}, c_{4}$ and $c_{5}$ are numbers larger than 0 . The electric current in the external resistive load is given by:

$$
\mathbf{n} \cdot \mathbf{J}=z_{i m p}\left(V-V_{f l}\right)
$$

where $z_{i m p}$ is the impedance of the resistive load $[\mathrm{m} / \mathrm{S}]$ and $V_{f l}$ is a reference electric potential [V]. The electric current in surfaces electrodes is given by:

$\mathbf{r} \cdot \mathbf{J}=\sigma_{p e r} V$

where $\sigma_{\text {per }}$ is the conductivity of the surface electrode $[\mathrm{S} / \mathrm{m}]$. The heat transfer due to convection is given by:

$\mathbf{n} \cdot \mathbf{Q}=h_{\text {conv }}\left(T-T_{f l}\right)$

where $h_{\text {conv }}$ is the convective heat transfer coefficient $\left[\mathrm{W} / \mathrm{m}^{2}\right]$ and $T_{f l}$ is the temperature of the ambient $[\mathrm{K}]$.

\section{Finite element formulation}

The topology optimization approach takes basis in the idea of spatially distributing two different material phases, $\Omega_{A}$ and $\Omega_{B}$ in a design space $\Omega_{D}$, in order to optimize for some performance measure. With basis in Eqs. (1)(4), $\Omega_{A}$ and $\Omega_{B}$ are initially clearly separated by a well-defined boundary $\Gamma$. The material phases represent two different TE active materials, Material A and Material $\mathrm{B}$. The equations are rewritten in a unified domain formulation, where no well-defined boundary between the material phases is required, by introducing a design variable field, $0 \leq \rho \leq 1$, so that Eqs. (1)-(4) become a functional of the design field, i.e. $\alpha(T)=\alpha(T, \rho)$, $\sigma(T)=\sigma(T, \rho)$ and $\kappa(T)=\kappa(T, \rho)$. A schematic of the concept of the unified domain, the design variable field and the corresponding boundary conditions in Eq. (5) have been sketched in Fig. 2. Elements with $\rho=0$ 
behave physically like Material A; elements with $\rho=1$ behave physically like Material B; and elements with $0<\rho<1$ are in an intermediate state between Material $\mathrm{A}$ and Material B. The thermal and electrical contact resistances in the transition between the materials are neglected.

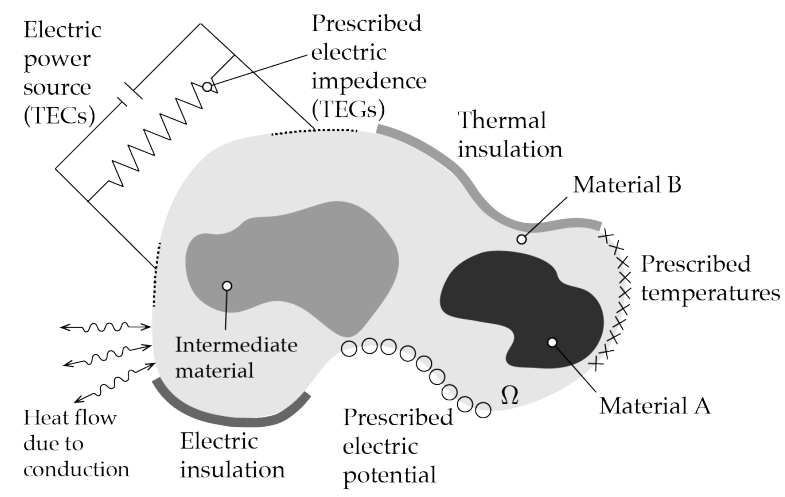

Fig. 2: A schematic of an arbitrary domain $\Omega$ relaxed by the design variable field $\rho$ and bounded by the boundary conditions stated in Eq. (5)

The discretized finite element equations are obtained by multiplying the strong forms of the equations in Eq. (1)-(4) with a suitable test function; integrating over the domain; performing integration by parts of higher dimensions on relevant terms; and introducing the design field dependent interpolation functions (Antonova and Looman, 2000, Cook et al., 2007, Yushanov et al., 2011).

The unified version of Eqs. (1)-(4) are discretised using bilinear quadrilateral finite elements with linear shape functions. The discrete variational problem is based on the Galerkin method where suitable finite dimensional solution spaces are introduced. Without further details, the discretized system of equations in Eqs. (1)-(4) are given by (Antonova and Looman, 2000, Cook et al., 2007):

$$
\begin{array}{r}
{\left[\begin{array}{cc}
\mathbf{K}^{T T}(\boldsymbol{\rho}, \mathbf{T})+\mathbf{H}^{T T} & 0 \\
\mathbf{K}^{V T}(\boldsymbol{\rho}, \mathbf{T}) & \mathbf{K}^{V V}(\boldsymbol{\rho}, \mathbf{T})+\mathbf{H}_{1}^{V V}+\mathbf{H}_{2}^{V V}
\end{array}\right]\left\{\begin{array}{c}
\mathbf{T} \\
\mathbf{V}
\end{array}\right\}} \\
=\left\{\begin{array}{c}
\mathbf{Q}^{P}(\mathbf{T}, \mathbf{V})+\mathbf{Q}^{E}(\mathbf{T}, \mathbf{V}) \\
\mathbf{0}
\end{array}\right\}
\end{array}
$$

where $\mathbf{K}^{T T}$ is the thermal stiffness matrix; $\mathbf{H}^{T T}$ is the heat transfer due to convection stiffness matrix; $\mathbf{K}^{V T}$ is the electric stiffness matrix; $\mathbf{K}^{V V}$ is the Seebeck stiffness matrix; $\mathbf{H}_{1}^{V V}$ is the electric resistance in outer load stiffness matrix; $\mathbf{H}_{2}^{V V}$ is the electric conductivity of the surface electrode stiffness matrix; $\mathbf{Q}^{P}$ is the Peltier heat load vector; and $\mathbf{Q}^{E}$ is the Joule heating load vector. Lower case letters denote generally element stiffness matrices and vectors, and capital letters denotes generally global stiffness matrices and vectors. The global system matrices and load vectors in Eqs. 11a are assembled from the local stiffness matrices with a standard finite element assembly procedure:

$\mathbf{K}^{T T}=\sum_{e=1}^{N} \mathbf{k}^{T T}, \quad \mathbf{H}^{T T}=\sum_{e=1}^{N} \mathbf{k}^{V T}, \quad \ldots$

The element system matrices are given by:

$$
\begin{aligned}
& \mathbf{k}^{T T}=\int_{\Omega} \mathbf{B}^{T} \boldsymbol{\kappa} \mathbf{B} \mathrm{d} V \\
& \mathbf{h}^{T T}=\int_{\Gamma} h_{c o n v} \mathbf{N}^{T} \mathbf{N} \mathrm{d} S \\
& \mathbf{k}^{V V}=\int_{\Omega} \mathbf{B}^{T} \boldsymbol{\sigma} \mathbf{B} \mathrm{d} V \\
& \mathbf{k}^{V T}=\int_{\Omega} \mathbf{B}^{T} \boldsymbol{\sigma} \boldsymbol{\alpha} \mathbf{B} \mathrm{d} V \\
& \mathbf{h}_{1}^{V V}=\int_{\Gamma} z_{i m p} \mathbf{N}^{T} \mathbf{N} \mathrm{d} S \\
& \mathbf{h}_{2}^{V V}=\int_{\Gamma} \sigma_{p e r} \mathbf{N}^{T} \mathbf{N} \mathrm{d} S \\
& \mathbf{q}^{P}=\int_{\Omega} \mathbf{B}^{T} T_{e} \boldsymbol{\alpha} \mathbf{J} \mathrm{d} V \\
& \mathbf{q}^{E}=\int_{\Omega} \mathbf{N}^{T} \mathbf{B}^{T} V_{e} \mathbf{J} \mathrm{d} V
\end{aligned}
$$

where $\boldsymbol{\alpha}$ is the Seebeck coefficient matrix; $\boldsymbol{\sigma}$ is the electric conductivity matrix; $\boldsymbol{\kappa}$ is the thermal conductivity matrix; $\mathbf{N}$ is the matrix of element shape functions; $\mathbf{B}$ is the derivative of $\mathbf{N}$, i.e. $\mathbf{B}=\nabla_{x} \mathbf{N}$. $T_{e}$ and $V_{e}$ are the element temperature and element electric potentials, respectively, and are given by:

$V_{e}=\mathbf{N}^{T} \mathbf{v}$
$T_{e}=\mathbf{N}^{T} \mathbf{t}$

where $\mathbf{t}$ and $\mathbf{v}$ are the nodal element temperatures and electric potentials. The element electric current density and the element heat flux can now be computed as:

$$
\begin{aligned}
\mathbf{J}(T, V) & =-\boldsymbol{\sigma} \mathbf{B}^{T} V_{e}-\boldsymbol{\sigma} \boldsymbol{\alpha} \mathbf{B}^{T} T_{e} \\
\mathbf{Q}(T, V) & =T_{e} \boldsymbol{\alpha} \mathbf{J}-\boldsymbol{\kappa} \mathbf{B}
\end{aligned}
$$


where the element material parameter matrices are given by:

$$
\begin{gathered}
\boldsymbol{\alpha}(\rho, T)=\left[\begin{array}{ll}
\alpha_{x x}(\rho, T) & \alpha_{x y}(\rho, T) \\
\alpha_{x y}(\rho, T) & \alpha_{y y}(\rho, T)
\end{array}\right] \\
\boldsymbol{\sigma}(\rho, T)=\left[\begin{array}{ll}
\sigma_{x x}(\rho, T) & \sigma_{x y}(\rho, T) \\
\sigma_{x y}(\rho, T) & \sigma_{y y}(\rho, T)
\end{array}\right] \\
\boldsymbol{\kappa}(\rho, T)=\left[\begin{array}{ll}
\kappa_{x x}(\rho, T) & \kappa_{x y}(\rho, T) \\
\kappa_{x y}(\rho, T) & \kappa_{y y}(\rho, T)
\end{array}\right]
\end{gathered}
$$

The material parameters are in this study assumed isotropic, so $\alpha_{x y}=\sigma_{x y}=\kappa_{x y}=0$. However, the framework can easily support anisotropic materials, simply by imposing non-zero values of the material parameters in the off-diagonal in Eqs. (16). The Seebeck coefficient, the electric conductivity and the thermal conductivity are interpolated between Material A and B by the following interpolation functions:

$\alpha_{i j}(\rho)=\frac{\kappa_{i j}^{A}(1-\rho) \alpha_{i j}^{B}+\kappa_{i j}^{B} \rho \alpha_{i j}^{A}}{\kappa_{i j}^{A}(1-\rho)+\kappa_{i j}^{B} \rho}$

$\sigma_{i j}(\rho)=\frac{\sigma_{i j}^{A} \sigma_{i j}^{B}}{\sigma_{i j}^{A}(1-\rho)+\sigma_{i j}^{B} \rho}$

$\kappa_{i j}(\rho)=\frac{\kappa_{i j}^{A} \kappa_{i j}^{B}}{\kappa_{i j}^{A}(1-\rho)+\kappa_{i j}^{B} \rho}$

where the indices $i$ and $j$ can take the values $x$ and $y$, compare with Eq. (16). The interpolation functions in Eqs. (17) describe the relationship between two materials in a segmented configuration in a $1 \mathrm{D}$ unit cell, for which reason the interpolation functions in Eq. (17) do not provide a physical interpretation of the intermediate designs in 2D and 3D. However, design designs take values in the extremes of Eq. (17) for which reason these designs are physically meaningful.

The residual equation of the discretized finite element equations in Eq. (9) is written as:

$\mathbf{R}=\mathbf{M}(\boldsymbol{\rho}, \mathbf{S}) \mathbf{S}-\mathbf{F}(\mathbf{S})=\mathbf{0}$

where $\mathbf{R}$ is the residual vector, $\mathbf{M}$ is the system matrix, $\mathbf{S}=\{\mathbf{T}, \mathbf{V}\}$ is the state variable vector and $\mathbf{F}$ is the load vector. The residual equation is solved by a combination between the undamped Newton's method (see e.g. Deuflhard (2014)) and Picard iterations.

\section{Topology optimization}

\subsection{Problem definition}

The optimization problem is formulated in a $\min / \max$ form for $k=\left\{1,2, \ldots, N^{k}\right\}$ projected realizations of the design variable field to ensure length-scale control and robustness toward manufacturing variations (Sigmund, 2009, Wang et al., 2011). The so-called robust formulation is given by:

$$
\begin{array}{ll}
\min _{\rho} & \max _{k}\left(f^{k}\right) \\
\text { s.t. } & \mathbf{R}^{k}\left(\overline{\tilde{\boldsymbol{\rho}}}^{k}, \mathbf{S}^{k}\right)=\mathbf{0} \\
& \mathbf{0} \leq \boldsymbol{\rho} \leq \mathbf{1} \quad \forall \boldsymbol{\rho} \in \Omega_{D}
\end{array}
$$

The optimization problem in Eq. (19) is solved for three realizations $N^{r}=3$, denoted the eroded, the nominal and the dilated designs, respectively. The nominal design variable field is plotted throughout in this paper.

\subsection{Adjoint sensitivities}

The gradients of the objective function with respect to the design variable field are required in order to solve the optimization problem in Eq. (19). The sensitivities of the $k^{\prime}$ th design realization, $\mathrm{d} L^{k} / \mathrm{d} \rho^{k}$, where $L$ is the general Lagrangian functional, are computed by the discrete adjoint approach (see Bendsøe and Sigmund (2003) and the references therein). The discrete adjoint approach requires the solution of the nonlinear forward problem in Eq. (18) and an additional linear adjoint problem:

$-\left(\nabla_{S^{k}} \mathbf{R}^{k}\right)^{T} \boldsymbol{\lambda}^{k}=\left(\nabla_{S^{k}} f^{k}\right)^{T}$

where $\boldsymbol{\lambda}^{k}$ is the vector of adjoint variables and $\square^{T}$ denotes the matrix or vector transpose operation. The term is Eq. (20) are evaluated for the solution of the forward problem. With reference to Eq. (20), the sensitivities can now be computed by the following expression:

$\frac{\mathrm{d} L^{k}}{\mathrm{~d} \boldsymbol{\rho}}=\frac{\mathrm{d} f^{k}}{\mathrm{~d} \boldsymbol{\rho}}=\nabla_{\rho} f^{k}-\left(\boldsymbol{\lambda}^{k}\right)^{T} \nabla_{\rho} \mathbf{R}^{\mathbf{k}}$

where $\boldsymbol{\rho}$ denotes the design variable vector, $\frac{\mathrm{d}}{\mathrm{d} \square}$ denotes the total derivative and $\nabla_{\square}$ denotes the partial derivative. Dropping the design realization notation, the tangent residual matrix, $\nabla_{S} \mathbf{R}$, in Eq. (20) is given by:

$\nabla_{S} \mathbf{R}=\mathbf{M}+\nabla_{S} \mathbf{M} \cdot \mathbf{S}-\nabla_{S} \mathbf{F}$

where

$\nabla_{S} \mathbf{M} \cdot \mathbf{S}=\left[\begin{array}{cr}\nabla_{T} \mathbf{K}^{T T} \cdot \mathbf{T} & \mathbf{0} \\ \nabla_{T} \mathbf{K}^{T V} \cdot \mathbf{T}+\nabla_{T} \mathbf{K}^{V V} \cdot \mathbf{V} & \mathbf{0}\end{array}\right]$

and

$\nabla_{S} \mathbf{F}=\left[\begin{array}{cc}\nabla_{T} \mathbf{Q}^{P}+\nabla_{T} \mathbf{Q}^{E} \nabla_{V} \mathbf{Q}^{P}+\nabla_{V} \mathbf{Q}^{E} \\ \mathbf{0} & \mathbf{0}\end{array}\right]$ 
Here, $\nabla_{T} \square$ and $\nabla_{V} \square$ denote the derivative of $\square$ with respect to $\mathbf{T}$ and $\mathbf{V}$, respectively. The dot product notation [.] between a matrix and a vector corresponds to sum over the nearest indices in tensor notation: If $\mathbf{A}$ is a matrix and $\mathbf{b}$ is a vector, then $\mathbf{A} \cdot \mathbf{x}$ is equivalent to $A_{i j} b_{j}$ in tensor notation. Notice that Eq. (22) is zero if the material parameters are assumed temperature independent. The tangent residual matrix with respect to the design variable field is given by:

$\nabla_{\rho} \mathbf{R}=\left[\begin{array}{c}\nabla_{\rho} \mathbf{K}^{T T} \cdot \mathbf{T}-\nabla_{\rho} \mathbf{Q}^{P}-\nabla_{\rho} \mathbf{Q}^{E} \\ \nabla_{\rho} \mathbf{K}^{V T} \cdot \mathbf{T}+\nabla_{\rho} \mathbf{K}^{V V} \cdot \mathbf{V}\end{array}\right]$

The adjoint load $\nabla_{S} f$ and the load in Eq. (21), $\nabla_{\rho} f$, depend on the objective function and these terms will be accounted for in relevant sections.

\subsection{Filters and Projection Strategy}

The physical design variables, $\overline{\tilde{\rho}}_{i}^{k}$, are used in the FE analysis and are obtained by the projection:

$\overline{\tilde{\rho}}_{i}^{k}=\frac{\tanh \left(\beta \eta^{k}\right)+\tanh \left(\beta\left(\tilde{\rho}_{i}-\eta^{k}\right)\right)}{\tanh \left(\beta \eta^{k}\right)+\tanh \left(\beta\left(1-\eta^{k}\right)\right)}$

where $\eta^{k}$ is the projection filter threshold. The filtered design variables $\tilde{\rho}_{i}$ are obtained from the mathematical design variables, $\rho_{i}$, by the filter operation:

$\tilde{\rho}_{i}=\frac{\sum_{j \in \mathbb{N}_{i}} w\left(\mathbf{x}_{j}\right) v_{j} \rho_{j}}{\sum_{j \in \mathbb{N}_{i}} w\left(\mathbf{x}_{j}\right) v_{j}}$

where $v_{j}$ is the area of the $j$ 'th element, $\mathbb{N}_{i}$ is the index set of the design variables which are within the radius $R$ of design variable $i, w(\mathbf{x})$ is the filter weighting function and $x_{i}$ and $x_{j}$ are the spatial location of elements $i$ and $j$. The filter weighting function is given by:

$w\left(\mathbf{x}_{j}\right)=\left\{\begin{array}{cc}R-|\mathbf{x}| & \forall|\mathbf{x}| \leq R \wedge \mathbf{x} \in \Omega_{D} \\ 0 & \text { otherwise }\end{array}\right.$

where $R$ is the filter radius, $|\mathbf{x}|=x_{i}-x_{j}$.

Finally, gradients with respect to design variables, $\rho_{i}$, require a transformation of the sensitivities by the chain rule:

$\frac{\partial L^{k}}{\partial \rho_{i}}=\sum_{j \in \Omega_{D}} \frac{\partial f^{k}}{\partial \tilde{\tilde{\rho}}_{j}^{k}} \frac{\partial \overline{\tilde{\rho}}_{j}^{k}}{\partial \tilde{\rho}_{j}} \frac{\partial \tilde{\rho}_{j}}{\partial \rho_{i}}$

\section{Implementation}

The finite element equations and sensitivities are derived in Maple and implemented in Matlab. The electric current density $\mathbf{J}$ and the thermal heat flux $\mathbf{Q}$ are evaluated in the centers of the elements. The finite element implementation has been verified with the commercial finite element software COMSOL and analytic derivations from Rowe (2005), Yang et al. (2012).

The optimization problems are solved using the method of moving asymptotes (Svanberg, 1987) with the standard settings and a move limit of 0.25 . The Heaviside projection parameter is updated every 50th design iteration after the scheme: $\beta=\{4,8,16,32,64,128\}$. The design process is stopped when $\beta=128$ and the design process is converged, i.e. when the max difference between the design variables in iteration $k$ and $k-1$ is less than $0.1 \%$.

The projection filter threshold values for the eroded, nominal and dilated designs are $\eta^{k}=\{0.3,0.5,0.7\}$, respectively. The density filter radius $R$ is chosen to provide a physical minimum length scale, relative to the design domain height, $L_{y}$, of 0.05 (see Wang et al. (2011) for more informations).

The robust topology optimization formulation ensures length-scale control and manufacturable designs, and the choice of interpolation functions provide, to our experience, well-posed and fast converging optimization problems.

\section{Numerical examples}

To demonstrate the capability and versatility of the density-based topology optimization approach presented in Sec. 4, we have solved and discussed seven different optimization problems for various boundary conditions, objective functions and material parameters. The numerical examples take basis in the schematic in Fig. 3 which illustrates an isolated leg of a TE module in Fig. 1b. The design domain, $\Omega_{D}$, is rectangular with length $L_{x}$ and height $L_{y}$. The northern, southern, eastern and western surfaces of the design domain are denoted $\Gamma^{N}$, $\Gamma^{S}, \Gamma^{E}, \Gamma^{W}$, respectively. The thermal energy entering $\Omega_{D}$ through $\Gamma^{N}, \Gamma^{S}, \Gamma^{E}$ and $\Gamma^{W}$ is modeled by convective heat transfer with convection coefficients $h_{\text {conv }}^{N}$, $h_{\text {conv }}^{S}, h_{\text {conv }}^{E}$ and $h_{\text {conv }}^{W}$ and reference temperatures $T_{f l}^{N}$, $T_{f l}^{S}, T_{f l}^{E}$ and $T_{f l}^{W}$, respectively. If convection coefficients $h_{\text {conv }}^{E}$ and $h_{\text {conv }}^{E}$ are equal they may be denoted $h_{\text {conv }}^{E W}$ to simplify the notation. An outer resistive load (for TEG problems) or an electric potential difference (for TEC problems) is applied between $\Gamma^{W}$ and $\Gamma^{E}$. The optimization problems are solved for $L_{x}=L_{y}=0.01$ and $\Omega_{D}$ is discretized into $100 \times 100$ finite elements. 


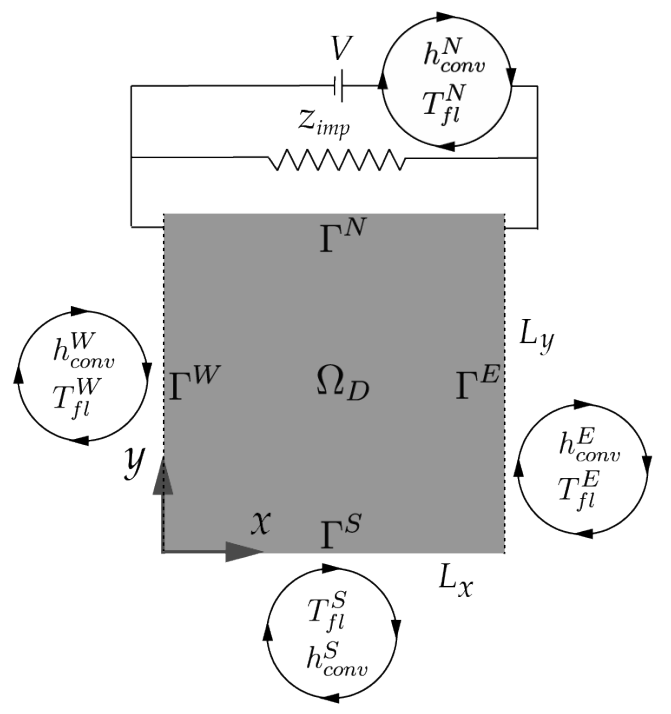

Fig. 3: Schematic of the TEG and TEC design problems

The approach presented in Secs. 3-4 supports arbitrary combinations of temperature dependent and independent material parameters, model dimensions and boundary conditions provided that the material parameters are in a range where the Newton solver is numerically stable. Three different sets of material parameters with different characteristics have been listed in Tab. 1. The constant material parameters are named after the authors of the papers in which the material parameters have been found. The color map used to present design solutions is chosen such that blue corresponds to Material A and yellow corresponds to Material B.

The relationships between $\alpha, \sigma, \kappa$ and $T$ for the temperature dependent material parameters have been plotted in Fig. 4. The temperature dependent materials are self-invented and do not refer to any physical materials. The blue curves are Material A and the yellow and black curves are Material B. Please notice the similarity between the colors of the curves and the colors of the materials phases in Tab. 1 and the design solutions. The relationships between $\alpha, \sigma, \kappa$ and $T$ are chosen such that complex interactions between the material parameters occur in the temperature range between 0 and $1000 \mathrm{~K}$. The temperature dependent material parameter set is purely academic and is primarily serving as a demonstration of the framework. However, physical realistic materials can easily be implemented if the polynomial relationships between the temperature and the material parameters are known.

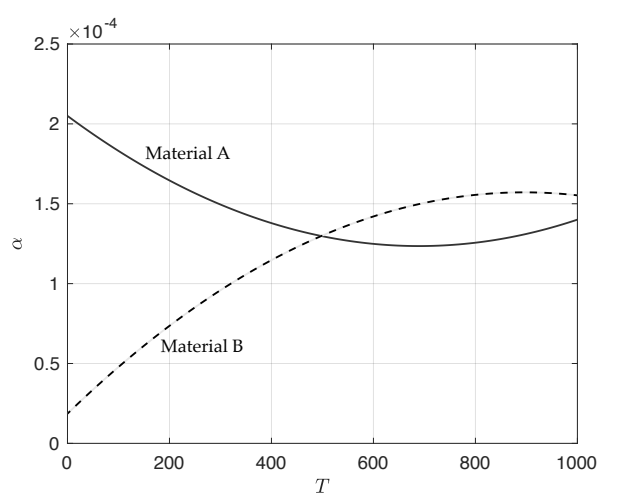

(a) Seebeck coefficient

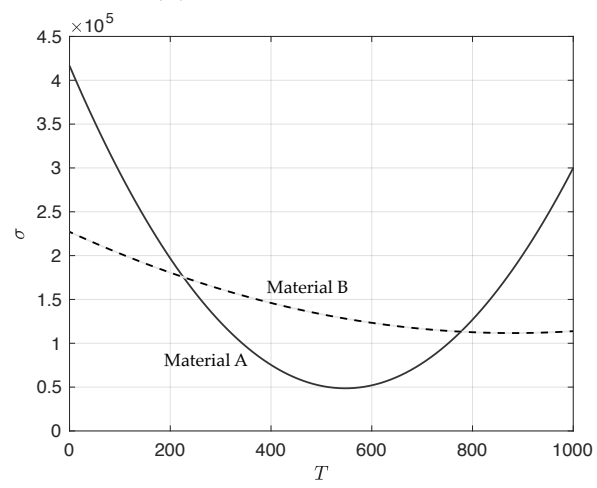

(b) Electric conductivity

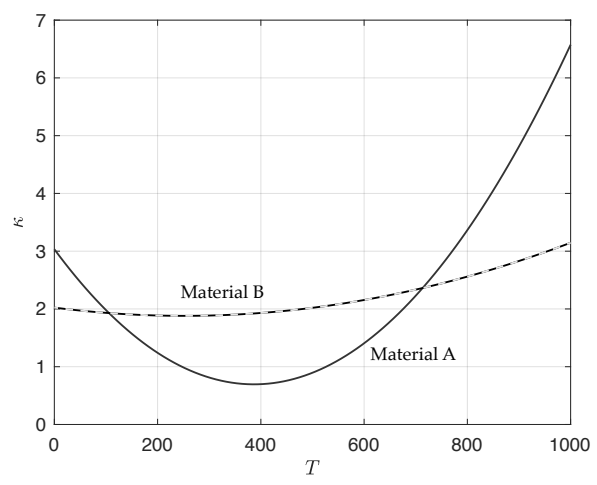

(c) Thermal conductivity

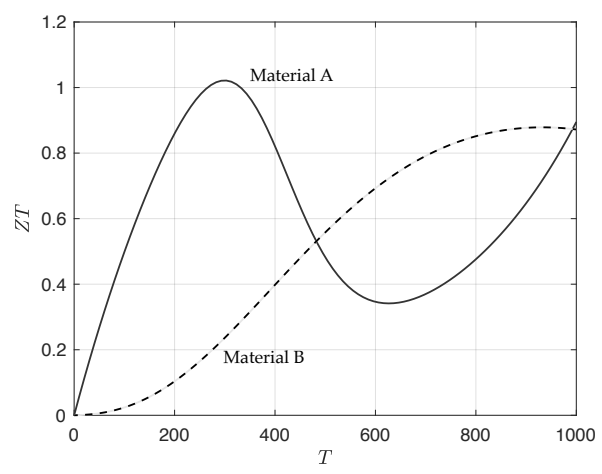

(d) Figure-of-merit

Fig. 4: The relationship between the temperature and the material parameters for the temperature dependent materials in Tab. 1 
Table 1: Material parameter sets used in the numerical examples

\begin{tabular}{|c|c|c|c|c|c|c|}
\hline Material parameter sets & & Color in plots & $\alpha[\mathrm{V} / \mathrm{K}]$ & $\sigma[\mathrm{S} / \mathrm{m}]$ & $\kappa[\mathrm{W} /(\mathrm{m} \cdot \mathrm{K})]$ & $Z[1 / \mathrm{K}]$ \\
\hline \multirow[t]{2}{*}{ Yang et al. (2012) } & MaterialA & & $200 \cdot 10^{-6}$ & $110 \cdot 10^{3}$ & 1.6 & $2.75 \cdot 10^{-3}$ \\
\hline & Material B & & $270 \cdot 10^{-6}$ & $22 \cdot 10^{3}$ & 0.77 & $\cdot 10^{-3}$ \\
\hline \multirow{2}{*}{$\begin{array}{l}\text { Temperature } \\
\text { dependent }\end{array}$} & Material A & & $\alpha_{A}(T)$ & $\sigma_{A}(T)$ & $\kappa_{A}(T)$ & $Z_{A}(T)$ \\
\hline & Material B & & $\alpha_{B}(T)$ & $\sigma_{B}(T)$ & $\kappa_{B}(T)$ & $Z_{B}(T)$ \\
\hline \multirow[t]{2}{*}{ Angst (2016) } & Material A & & $-200 \cdot 10^{-6}$ & $100 \cdot 10^{3}$ & 2.0 & $2.0 \cdot 10^{-3}$ \\
\hline & Material B & & $200 \cdot 10^{-6}$ & $100 \cdot 10^{3}$ & 2.0 & $2.0 \cdot 10^{-3}$ \\
\hline
\end{tabular}

The convection coefficients for various flow types and flow conditions have been listed in Tab. 2. These values are basis for the convection boundary conditions of the design problems discussed in the following sections.

In the following sections we will consider seven different optimization problems: In Sec. 6.1.1-6.1.2 we optimize TEGs for electric power output and conversion efficiency. We optimize TECs for temperature in Sec. 6.2.1, for heat flux in Sec. 6.2.2 and for coefficient of performance in 6.2.3. In Sec. 6.3 we optimize TEGs for electric power output and conversion efficiency for asymmetric boundary conditions. Finally, in Sec. 6.4 we investigates an electric power output design problem for so-called $p$ - $n$ generators.

\subsection{Thermoelectric generators}

We aim at optimizing for two different objective functions for TEGs: The electric power output, $f_{P}$, and the electric conversion efficiency, $f_{\eta}$. The boundary conditions for the $f_{P}$ problem are summarized in Tab. 3 . The problem setup is inspired by waste heat recovery applications in e.g. power plants, where designers aim at maximizing electric power production by utilizing the thermal energy exchange between hot exhaust gas and the cold ambient. $h_{\text {conv }}^{E}$ and $h_{\text {conv }}^{W}$ control the magnitude of the thermal input available in the hot and the cold reservoirs. If $h_{c o n v}^{E W}=0$ there is no thermal energy available. If $h_{\text {conv }}^{E W}=\infty$ (equivalent to fixed boundary conditions) there is an infinite amount of energy available. The convection coefficients depend on the flow types on $\Gamma^{E}$ and $\Gamma^{W}$, however physical convection coefficients are somewhere in between these (nonphysical) extremes, compare with Tab. 2. A comprehensive review of heat transfer mechanics in TE materials and devices is discussed in Tian et al. (2014).

\subsubsection{Electric power output}

The first numerical example aims at optimizing the electric power output, $f_{P}$, by converting the thermal heat inputs on $\Gamma^{E}$ and $\Gamma^{W}$ into electric energy. TEGs are similar to batteries in electric circuits: To maximize the electric power output, it is necessary to match the internal and external resistance of the TEG. The electric power output objective is in weak form given by:

$f_{P}=\frac{1}{L_{y}} \int_{\Gamma^{E}} V \mathrm{~d} S \int_{\Gamma^{E}} J_{x} \mathrm{~d} S$

which can be rewritten in what we call finite element form as:

$f_{P}=\left(\sum_{i \in \mathbb{N}_{E}} \frac{1}{L_{y, i}} V_{i}\right) \sum_{j \in \mathbb{M}_{E}} J_{j}$

where $\mathbb{N}_{E}$ is the index sets of the nodes on $\Gamma^{E}$ and $\mathbb{M}_{E_{x}}$ is the $x$-directional index sets of the $x$ and $y$ directions of the centers of the elements on $\Gamma^{E}$. By introduction of the vectors $\mathbf{L}_{\mathbb{N}}^{T}$ and $\mathbf{L}_{\mathbb{M}}^{T}$, Eq. (31) can be written in the following form:

$f_{P}=\left(\mathbf{L}_{\mathbb{N}}^{T} \mathbf{V}\right)\left(\mathbf{L}_{\mathbb{M}}^{T} \mathbf{J}\right)$

where $\mathbf{L}_{\mathbb{N}}^{T}$ is a vector consisting of zeroes except for the positions $i \in \mathbb{N}_{E}$ which have the value $1 / N_{E}$ where $N_{E}$ is the number of nodes on $\Gamma^{E} . \mathbf{L}_{\mathbb{M}}^{T}$ is a vector consisting of zeroes except for the positions $i \in \mathbb{M}_{E}$ which have the value $1 / l_{y, i}$, where $l_{y, i}$ is the height of the $i$ 'th element. Objective functions written in the form such as in Eq. (31) are referred to as the implementation form for the rest of the paper. To solve the adjoint problem in Eqs. (20), we need the gradients of the objective function with respect to the design field, $\nabla_{\rho} f_{P}$, which can be computed as:

$\nabla_{\rho} f_{P}=\left(\mathbf{L}_{\mathbb{N}}^{T} \mathbf{V}\right)\left(\mathbf{L}_{\mathbb{M}}^{T} \nabla_{\rho} \mathbf{J}\right)$

Computing the gradients of the objective function with respect to the state field, $\nabla_{S} f_{P}$, provides at this instance all terms in Eq. (21). $\nabla_{S} f_{P}$ is given by: 
Table 2: $h_{\text {conv }}$ for various flow types and flow conditions

\begin{tabular}{llr}
\hline Flow type & Flow condition & $h_{\text {conv }}$ \\
\hline Forced convection & Low speed flow of air over a surface & 10 \\
& Moderate speed flow of air over a surface & 100 \\
& Moderate speed flow of air over a cylinder & 200 \\
& Moderate speed flow of water in a pipe & 3000 \\
& Molten metals & $2000-45000$ \\
& Boiling water in a pipe & 50000 \\
& Water and liquids & $50-10000$ \\
\hline Free convection & Water and liquids & $50-3000$ \\
& Water in free convection & $100-1200$ \\
& Air & $10-100$ \\
& Various gasses & $5-37$ \\
& Vertical plate in air & 5 \\
\hline
\end{tabular}

Table 3: Boundary conditions for the $f_{P}$ and $f_{\eta}$ TEG problem

\begin{tabular}{lcccc}
\hline & \multicolumn{4}{c}{ Boundary } \\
\cline { 2 - 5 } & $\Gamma^{N}$ & $\Gamma^{S}$ & $\Gamma^{E}$ & $\Gamma^{W}$ \\
\hline$T_{f l}$ & - & - & 1000 & 0 \\
$h_{\text {conv }}$ & - & - & $h_{\text {conv }}^{E}$ & $h_{\text {conv }}^{W}$ \\
$V$ & - & - & - & 0 \\
$\sigma_{\text {per }}$ & - & - & - & $10^{10}$ \\
$z_{\text {imp }}$ & - & - & - & $z_{i m p}$ \\
\hline
\end{tabular}

$\nabla_{S} f_{P}=\left\{\begin{array}{c}\left(\mathbf{L}_{\mathbb{N}}^{T} \mathbf{V}\right)\left(\mathbf{L}_{\mathbb{M}}^{T} \nabla_{T} \mathbf{J}\right) \\ \left(\mathbf{L}_{\mathbb{N}}^{T} \mathbf{V}\right)\left(\mathbf{L}_{\mathbb{M}}^{T} \nabla_{V} \mathbf{J}\right)+\mathbf{L}_{\mathbb{N}}^{T} \circ\left(\mathbf{L}_{\mathbb{M}}^{T} \mathbf{J}\right)\end{array}\right\}$

where $\circ$ denotes the Hadamard product (element wise multiplication).

The $f_{P}$ optimized designs for various $h_{c o n v}^{E W}$ have been plotted in Fig. 5. The design solutions are based on the temperature dependent material parameters in Tab. 1 and Fig. 4. The design solutions are indeed dependent on $h_{\text {conv }}^{E W}$ : Low magnitudes of $h_{\text {conv }}^{E W}$ result in "spike-shaped" transitions between the two material phases. Large magnitudes of $h_{c o n v}^{E W}$ result in abrupt transitions between the two material phases. The spike-shaped design features enable the designs to perform in an intermediate state between the two design phases. Design problems solved for large $h_{c o n v}^{E W}$ prefer a relatively larger amount of Material B compared to design problems solved for low $h_{c o n v}^{E W}$. The design optimized for $h_{c o n v}^{E W}=10000$ has two transitions between the two material phases. This design feature is caused by Material B's large magnitude of $\alpha$ for large $T$, confer Fig. 4. To provide additional insight, Fig. 6 plots the relationships between the $y$-directional average of the temperature along the spatial direction, $x$ for the design solutions in Fig. 5. The figure shows the relationships between $h_{\text {conv }}^{E W}$ and the temperature fields. The temperature difference between $\Gamma^{E}$ and $\Gamma^{W}$ is controlled by the magnitude of $h_{c o n v}^{E W}$, where a large $h_{c o n v}^{E W}$ yield a large temperature difference and vice versa. Increasing $h_{\text {conv }}^{E W}$ causes the temperatures on $\Gamma^{E}$ and $\Gamma^{W}$ to approach $T_{f l}^{E}$ and $T_{f l}^{W}$, respectively.

The impedance in the resistive load, $z_{i m p}$, is matched with the internal resistance of the designs to provide the largest possible $f_{P}$ and $f_{\eta}$. The design solutions depend on $z_{i m p}$ for which reason this design parameter is critical to include in the optimization problems.

Finally, it is important to state that the designs are physical realizable because the designs are optimized to $0 / 1$ solutions.

\subsubsection{Conversion efficiency}

In the second numerical example we aim at optimizing the TE conversion efficiency, $f_{\eta}$. The boundary conditions and material parameters for this problem are similar to the boundary conditions and material parameters for the $f_{P}$ problem in Sec. 6.1.1. The electric conversion efficiency is in implementation form given by:

$f_{\eta}=\frac{\left(\mathbf{L}_{\mathbb{N}}^{T} \mathbf{V}\right)\left(\mathbf{L}_{\mathbb{M}}^{T} \mathbf{J}\right)}{\mathbf{L}_{\mathbb{M}}^{T} \mathbf{Q}}$

Fraction symbols are in implementation notation interpreted as elementwise vector division. With reference to Eqs. (20) and (21), $\nabla_{\rho} f_{\eta}$ and $\nabla_{S} f_{\eta}$ can now be computed as: 


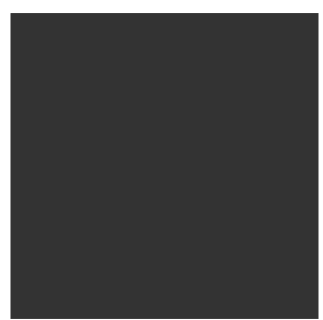

(a) $h_{\text {conv }}^{E W}=40$

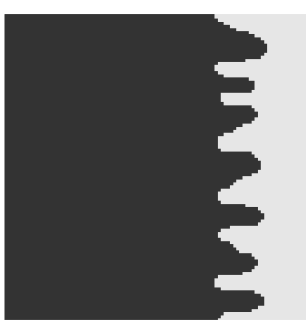

(b) $h_{\text {conv }}^{E W}=70$

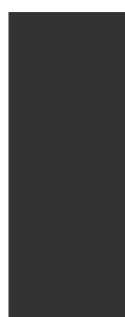

(c) $h_{\text {conv }}^{E W}=160$

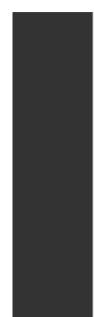

(d) $h_{c o n v}^{E W}=500$

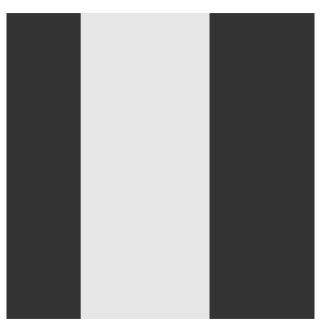

(e) $h_{\text {conv }}^{E W}=10000$

Fig. 5: Design solutions for $f_{P}$ objectives with tempature dependent material parameters in Tab. 1

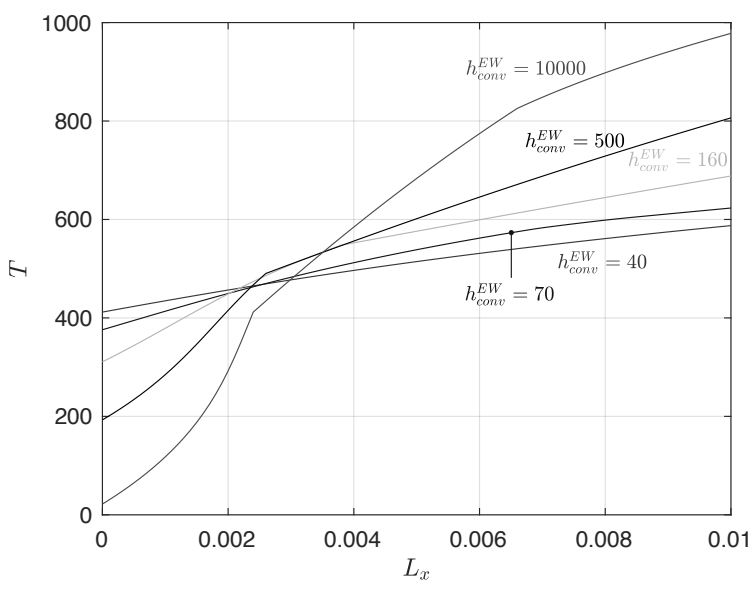

Fig. 6: The relationship between the average temperature along $\{x, y\}=\{x, y\}$

$$
\begin{aligned}
\nabla_{\rho} f_{\eta}= & \frac{\left(\mathbf{L}_{\mathbb{N}}^{T} \mathbf{V}\right)\left(\mathbf{L}_{\mathbb{M}}^{T} \nabla_{\rho} \mathbf{J}\right)}{\mathbf{L}_{\mathbb{M}}^{T} \mathbf{Q}} \\
& -\frac{\left(\mathbf{L}_{\mathbb{N}}^{T} \mathbf{V}\right)\left(\mathbf{L}_{\mathbb{M}}^{T} \mathbf{J} \nabla_{\rho} \mathbf{Q}\right)}{\mathbf{L}_{\mathbb{M}}^{T}(\mathbf{Q} \circ \mathbf{Q})} \\
\nabla_{S} f_{\eta}= & \left\{\begin{array}{c}
\frac{\left(\mathbf{L}_{\mathbb{N}}^{T} \mathbf{V}\right)\left(\mathbf{L}_{\mathbb{M}}^{T} \nabla_{T} \mathbf{J}\right)}{\mathbf{L}_{\mathbb{M}}^{T} \mathbf{Q}} \\
-\frac{\left(\mathbf{L}_{\mathbb{N}}^{T} \mathbf{V}\right)\left(\mathbf{L}_{\mathbb{M}}^{T} \mathbf{J} \nabla_{T} \mathbf{Q}\right)}{\mathbf{L}_{\mathbb{M}}^{T}(\mathbf{Q} \circ \mathbf{Q})} \\
\frac{\mathbf{L}_{\mathbb{N}}^{T} \circ\left(\mathbf{L}_{\mathbb{M}}^{T} \mathbf{J}\right)}{\mathbf{L}_{\mathbb{M}}^{T} \mathbf{Q}} \\
+\frac{\left(\mathbf{L}_{\mathbb{N}}^{T} \mathbf{V}\right)\left(\mathbf{L}_{\mathbb{M}}^{T} \nabla_{V} \mathbf{J}\right)}{\mathbf{L}_{\mathbb{M}}^{T} \mathbf{Q}} \\
-\frac{\left(\mathbf{L}_{\mathbb{N}}{ }^{T} \mathbf{V}\right)\left(\mathbf{L}_{\mathbb{M}}^{T} \mathbf{J} \nabla_{V} \mathbf{Q}\right)}{\mathbf{L}_{\mathbb{M}}^{T}(\mathbf{Q} \circ \mathbf{Q})}
\end{array}\right\}
\end{aligned}
$$

The design solution for the $f_{\eta}$ design problem have been plotted in Fig. 7. Two design features are similar to the $f_{P}$ design problem in Sec. 6.1.1: The spike-shaped transitions between the material phases for low $h_{c o n v}^{E W}$ and the design solution dependency of $h_{c o n v}^{E W}$. The two transitions between the two material phases for $h_{c o n v}^{E W}=$ 10000 are not observed for this optimization problem, which may be explained by the high magnitude of $\kappa^{B}$ for high magnitudes of $T$, confer Fig. 4. The high $\kappa^{B}$ for high $T$ decreases the effective thermal conductivity of the design which allow passage of more thermal heat from $\Gamma^{W}$ to $\Gamma^{E}$ which makes the double material phase for the $f_{\eta}$ objective cost inefficient.

The relationship between the normalized conversion efficiency $f_{\eta} /\left(\left.f_{\eta}\right|_{k=1}\right)$ and iteration number, $k$, for the design solution in Fig. 7b has been plotted in Fig. 8. Snapshots of the corresponding design evolution have been plotted in Fig. 9. We notice that the convergence of the design problem is smooth and stable despite the non-linear coupled physics and the temperature dependent material parameters. The "convex" behavior of the design problem may be explained by well-tuned optimization and model parameters, well-chosen interpolation functions and the diffusion-type nature of the governing physics. By comparing the convergence plot and the design evolution in Fig. 8-9, we notice that the difference in objection functions between the spikeshaped designs the abrupt transitions designs is small for this particular example.

\subsection{Thermoelectric coolers}

In TEC problems, we consider three different objective functions: the temperature average, $f_{T}$, the heat flux, $f_{Q}$, and the coefficient of performance (COP), $f_{\mu}$. The problem setup is inspired by a household refrigerator and takes basis in the design problem sketched in Fig. 3. The boundary conditions are listed in Tab. 4. A TEC is utilized to transfer energy from the thermal cold reservoir on $\Gamma^{E}$ to the themal hot reservoir on $\Gamma^{W}$ by 


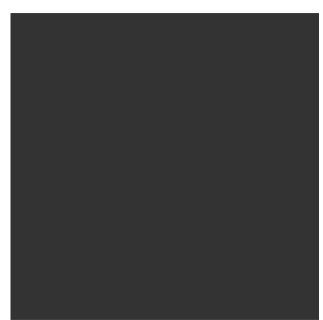

(a) $h_{c o n v}^{E W}=100$

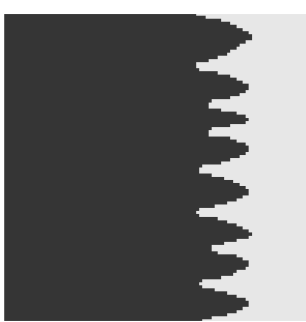

(b) $h_{\text {conv }}^{E W}=180$

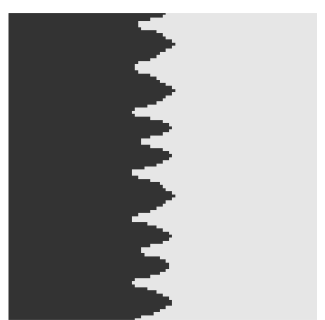

(c) $h_{\text {conv }}^{E W}=400$

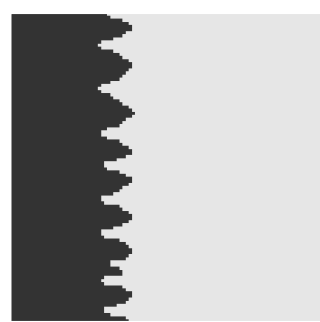

(d) $h_{\text {conv }}^{E W}=1000$

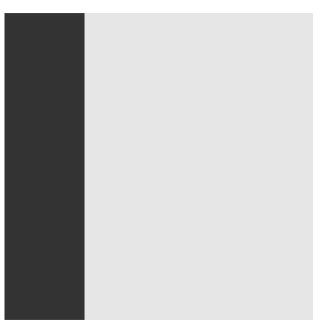

(e) $h_{\text {conv }}^{E W}=10000$

Fig. 7: Design solutions for $f_{\eta}$ objectives with tempature dependent material parameters in Tab. 1

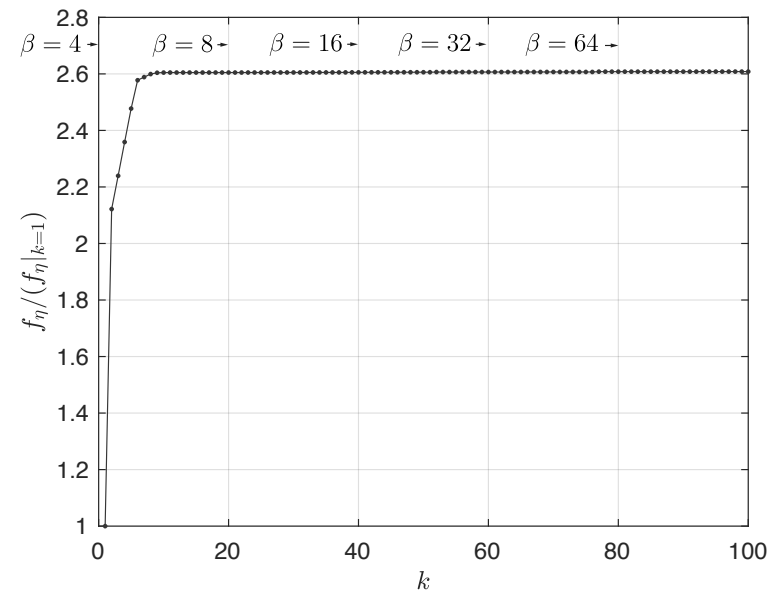

Fig. 8: Convergence plot for the design solution in Fig. $7 \mathrm{~b}$

Table 4: Boundary conditions for the $f_{T}, f_{Q}, f_{\mu}$ optimization problems

\begin{tabular}{lcccc}
\hline & \multicolumn{4}{c}{ Boundary } \\
\cline { 2 - 5 } & $\Gamma^{N}$ & $\Gamma^{S}$ & $\Gamma^{E}$ & $\Gamma^{W}$ \\
\hline$T_{f l}$ & - & - & 300 & 270 \\
$h_{\text {conv }}$ & - & - & $h_{\text {conv }}^{E}$ & $h_{\text {conv }}^{W}$ \\
$V$ & - & - & $V$ & 0 \\
$\sigma_{\text {per }}$ & - & - & $10^{10}$ & - \\
$z_{\text {imp }}$ & - & - & - & - \\
\hline
\end{tabular}

converting the electric energy imposed via an electric potential difference between $\Gamma^{E}$ and $\Gamma^{W}$ into cooling energy on $\Gamma^{E}$. The available energy in the hot and cold reservoirs is controlled by the magnitude of $h_{\text {conv }}^{E}$ and $h_{\text {conv }}^{W}$. With reference to Fig. $3, h_{\text {conv }}^{E}=0$ is equivalent as cooling into a completely insulated boundary. $h_{\text {conv }}^{E}=\infty$ is equivalent as cooling into an infinitely large heat reservoir. A convection coefficient in both of these extremes are nonphysical. Some physically realistic convection coefficients have been listed in Tab. 2 for comparison.

\subsubsection{Temperature}

The third optimization problem aims at optimizing $f_{T}$ objective which in implementation form is given by:

$f_{T}=\mathbf{L}_{\mathbb{N}}^{T} \mathbf{T}$

With reference to Eqs. (20) and (21), $\nabla_{\rho} f_{T}$ and $\nabla_{S} f_{T}$ can now be computed as:

$\nabla_{\rho} f_{T}=\mathbf{0}$
$\nabla_{S} f_{T}=\left\{\begin{array}{c}\mathbf{L}_{\mathbb{N}}^{T} \\ \mathbf{0}\end{array}\right\}$

The design solutions for the $f_{T}$ optimization problem optimized for the Yang et al. (2012) material parameters and various magnitudes of $h_{c o n v}^{E W}$ have been plotted in Fig. 10. The design features are generally similar to what we observed in Secs. 6.1.1 and 6.1.2. However, there are two details which defer slightly from the governing design features of the $f_{P}$ and $f_{\eta}$ design solutions: The spike-shaped transitions between the material phases are extended over a larger part of the design domain, and the transitions between the material phases occur for different $h_{\text {conv }}$.

To obtain the "optimal" $f_{T}$ for a given design problem, the electric energy input needs to be matched such that a compromise between the Peltier effect and the Joule heating is found. A cost ineffective high amount of internal Joule heating is generated for too high electric power inputs. A cost ineffective low amount of Peltier work is generated for too low electric power inputs. To demonstrate this compromise, the relationship between $V$ and $f_{T}$ for the design optimized for $h_{c o n v}^{E W}=400$ has been plotted in Fig. 11.

To determine how much significance we can attribute to the optimized designs, we have crosschecked the relationship between $f_{T}$ and $h_{c o n v}^{E W}$ for the designs in Fig. 10. All entries in the figure are evaluated for the optimal choice of electric potential difference between $\Gamma^{E}$ and $\Gamma^{W}$. The figure shows that a design optimized for one 


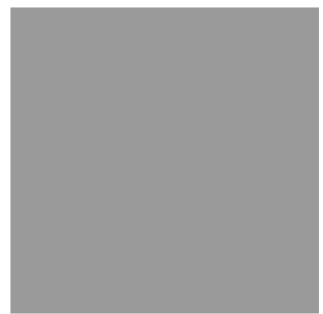

(a) $k=1$

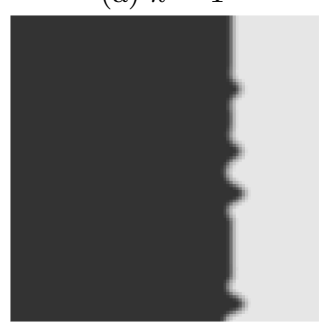

(f) $k=30$

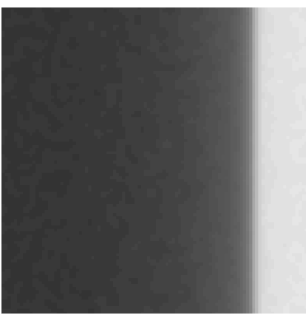

(b) $k=3$

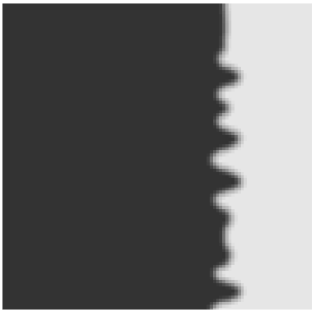

(g) $k=40$

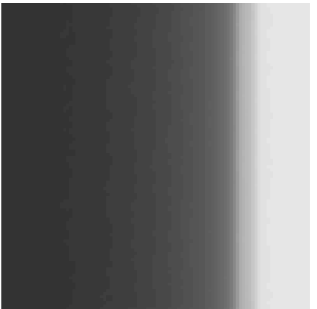

(c) $k=5$

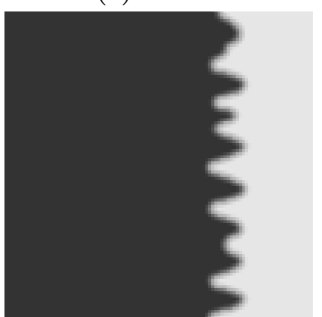

(h) $k=50$

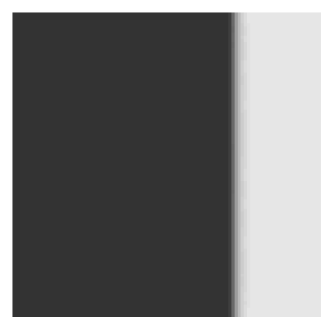

(d) $k=10$

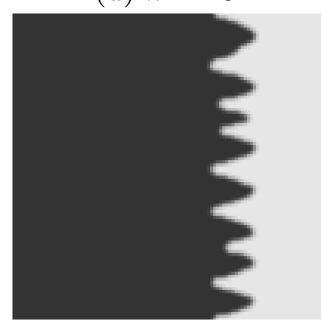

(i) $k=70$

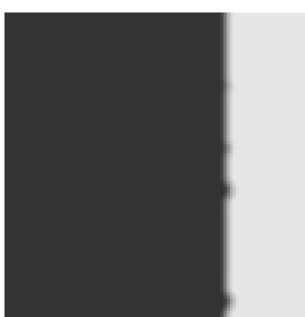

(e) $k=20$

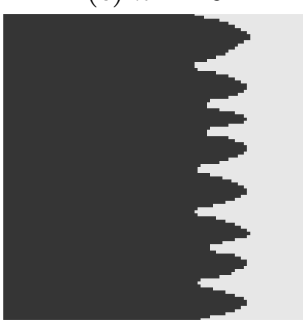

(j) $k=100$

Fig. 9: Design evolution for the $f_{\eta}$ optimization problem with $h_{c o n v}^{E W}=180$ and the temperature dependent material parameters

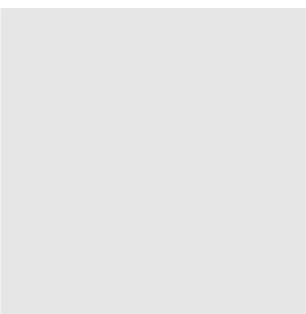

(a) $h_{\text {conv }}^{E W}=200$

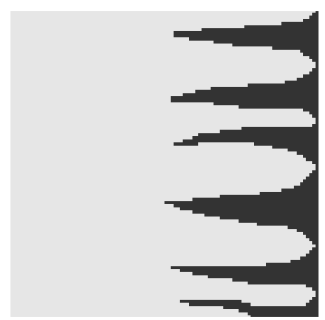

(b) $h_{\text {conv }}^{E W}=300$

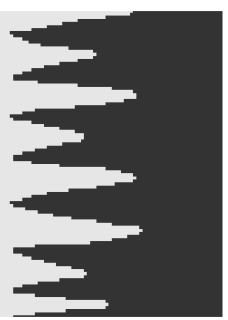

(c) $h_{\text {conv }}^{E W}=400$

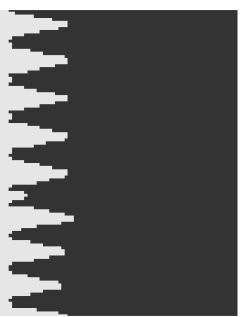

(d) $h_{\text {conv }}^{E W}=500$

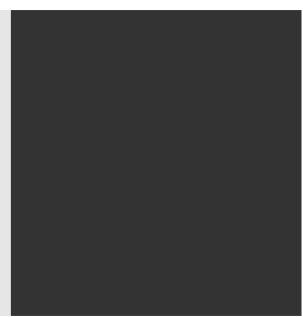

(e) $h_{\text {conv }}^{E W}=1000$

Fig. 10: Optimized designs for the $f_{T}$ optimization problem and Yang et al. (2012) material parameters

$h_{c o n v}^{E W}$ indeed has superior performance compared to designs optimized for an other $h_{c o n v}^{E W}$. Similar crosschecks have been performed and confirmed for all optimization problems presented in this study, however these studies have been omitted for space reasons.

\subsubsection{Heat flux}

The fourth numerical problem concerns the optimization of $f_{Q}$ which in implementation form is given by:

$f_{Q}=\mathbf{L}_{\mathbb{M}}^{T} \mathbf{Q}$

With reference to Eqs. (20) and (21), $\nabla_{\rho} f_{Q}$ and $\nabla_{S} f_{Q}$ can now be computed as:

$\nabla_{\rho} f_{Q}=\mathbf{L}_{\mathbb{M}}^{T} \nabla_{\rho} \mathbf{Q}$

$\nabla_{S} f_{T}=\left\{\begin{array}{l}\mathbf{L}_{\mathbb{M}}^{T} \nabla_{T} \mathbf{Q} \\ \mathbf{L}_{\mathbb{M}}^{T} \nabla_{V} \mathbf{Q}\end{array}\right\}$

The design solutions for the $f_{Q}$ optimization problem for various magnitudes of $h_{\text {conv }}$ have been plotted in Fig.
13. The spike-shaped transitions seem to be propagating over a shorter distance and the transitions between the material phases occur at different $h_{\text {conv }}$ compared to the designs in Secs. 6.1.1 and 6.1.2.

In Fig. 13 we find bands in the small band of Material A placed at $\Gamma^{E}$ for all magnitudes of $h_{c o n v}^{E W}$. The design feature occurs because $\mathbf{Q}$ is related to $\nabla_{x} \mathbf{T}$, and Material A has a relatively large $\alpha$ compared to Material $\mathrm{B}$, which combined adds a contribution to $\mathbf{Q}$ and hereby a cost effective contribution to $f_{Q}$. The design feature is from now on referred to as the band design feature.

\subsubsection{Coefficient of performance}

In the fifth numerical example we investigate $f_{\mu}$ which in implementation form is given by:

$f_{\mu}=\frac{\mathbf{L}_{\mathbb{M}}^{T} \mathbf{Q}}{\left(\mathbf{L}_{\mathbb{N}}^{T} \mathbf{V}\right)\left(\mathbf{L}_{\mathbb{M}}^{T} \mathbf{J}\right)}$

Eq. (42) is simply the inverse of $f_{\eta}$ in Eq. (35) for which reason $\nabla_{\rho} f_{\mu}$ and $\nabla_{x} f_{\mu}$ are easily obtainable from 


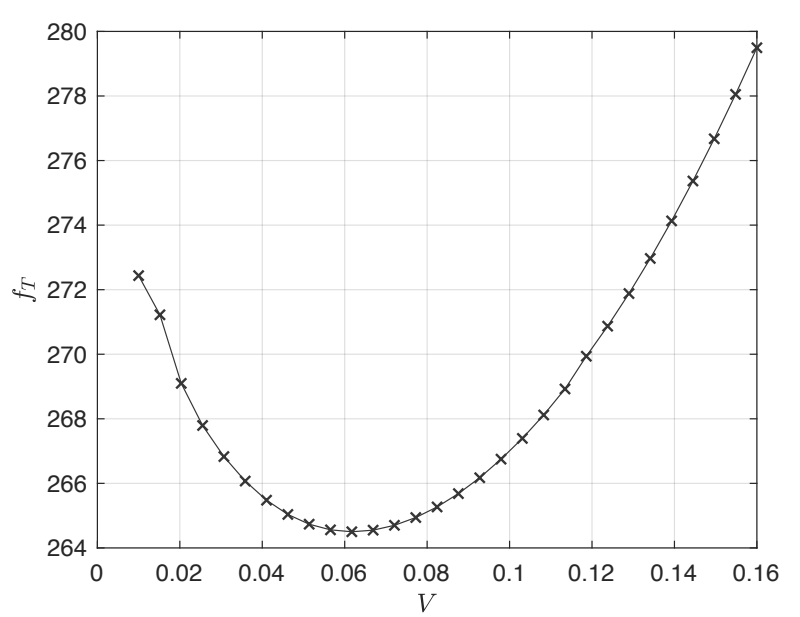

Fig. 11: Relationship between the electric potential different between $\Gamma^{E}$ and $\Gamma^{W}$ and the temperature on $\Gamma^{E}$ for the $f_{T}$ design optimized for $h_{c o n v}^{E W}=400$

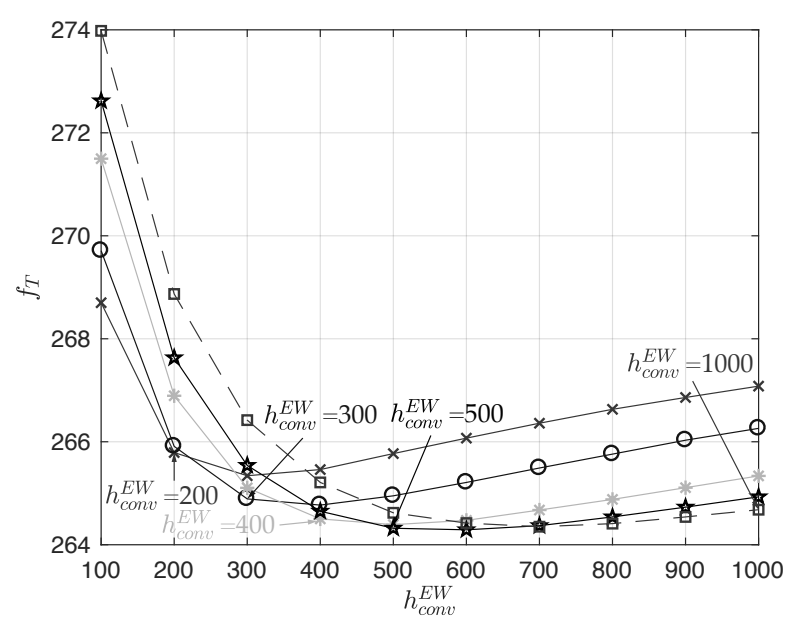

Fig. 12: Cross check between $f_{T}$ and $h_{\text {conv }}^{E W}$ for the designs in Fig. 10

Eq. (36). The $f_{\mu}$ design problem aims at extracting as much thermal energy as possible from the cold reservoir with the least amount of electric power usage. To avoid trivial design solutions, i.e. designs optimized for $V=0$, we have decided that the electric potential difference between $\Gamma^{E}$ and $\Gamma^{W}$ for $f_{\eta}$ problems is $V=0.0617$. This electric potential difference is equivalent to the best performing $f_{T}$ design optimized for $h_{c o n v}^{E W}=400$, see Fig. 11.

The $f_{\mu}$ design solutions for various $h_{\text {conv }}^{E W}$ have been plotted in Fig. 14. The band design feature is observed for all $h_{\text {conv }}^{E W}$. A new design feature is observed for $h_{\text {conv }}^{E W}=\{5000,8000\}$ where Material B is expanding out from $\Gamma^{E}$ in a trapezoid shaped topology.

By visual comparison between Figs. 10, 13 and 14 we notice that very different design solutions occur for these
Table 5: Boundary conditions for the asymmetric boundary condition problems

\begin{tabular}{llcccc}
\hline & & \multicolumn{4}{c}{ Boundary } \\
\cline { 3 - 6 } & & $\Gamma^{N}$ & $\Gamma^{S}$ & $\Gamma^{E}$ & $\Gamma^{W}$ \\
\hline Set 1 & $T_{f l}$ & 1000 & 330 & 1000 & 0 \\
& $h_{\text {conv }}$ & $10^{4}$ & $4.5 \cdot 10^{4}$ & $4.5 \cdot 10^{4}$ & $10^{4}$ \\
& $V$ & - & - & $V$ & 0 \\
& $\sigma_{\text {per }}$ & - & - & $10^{10}$ & - \\
& $z_{\text {imp }}$ & - & - & - & $z_{i m p}$ \\
\hline Set 2 & $T_{\text {fl }}$ & 1000 & 0 & 1000 & 0 \\
& $h_{\text {conv }}$ & $10^{5}$ & $10^{5}$ & $10^{5}$ & $10^{5}$ \\
& $V$ & - & - & $V$ & 0 \\
& $\sigma_{\text {per }}$ & - & - & $10^{10}$ & - \\
& $z_{\text {imp }}$ & - & - & - & $z_{i m p}$ \\
\hline
\end{tabular}

three different - however related - objective functions. It is therefore indeed critical to consider the end goal applications and the boundary conditions when designing TECs. To explain and fully understand the appearance of the trapezoid shaped design features in $f_{\mu}$ design problems and the spike shaped design features in $f_{T}$ and $f_{Q}$ design problems is an important and potential rewarding subject. However, that particular study goes far beyond the scope of this work and we are currently working on a dedicated TEC paper aiming to explain these design features in details.

\subsection{Asymmetric boundary conditions}

$1 D$ boundary conditions problems refer in this paper to problems where the temperature and electric potential boundary conditions are imposed only on parallel boundaries, such as $\Gamma^{E}$ and $\Gamma^{W}$. Asymmetric boundary conditions problems refer to problems where the boundary conditions are imposed on perpendicular and parallel boundaries, such as $\Gamma^{N}, \Gamma^{S}, \Gamma^{E}$ and $\Gamma^{W}$. The designs presented so far in Secs. 6.1-6.2 have been limited to $1 \mathrm{D}$ boundary conditions. Asymmetric boundary conditions may yield a deeper solutions space of the design problems, which may constitute more topological complex design solutions. Design solutions optimized for asymmetric boundary conditions are not likely to produce more efficient designs that the one dimensional boundary conditions. However, for physical or manufacturing reasons such designs may be desirable despite interior performance. The sixth numerical example concerns asymmetric boundary conditions and the design problem is primarily serving as an example of the versatility and application of the approach. We use Yang et al. (2012) material parameters, and consider the two sets of boundary conditions listed in Tab. 5 . 


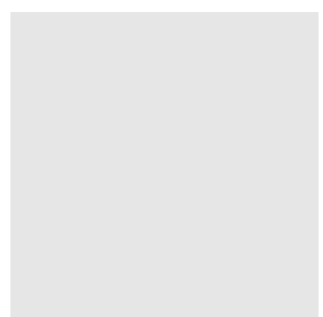

(a) $h_{\text {conv }}^{E W}=100$

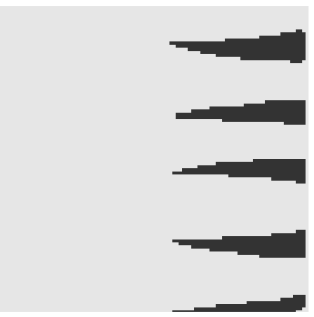

(b) $h_{\text {conv }}^{E W}=225$

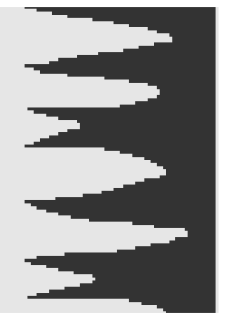

(c) $h_{\text {conv }}^{E W}=325$

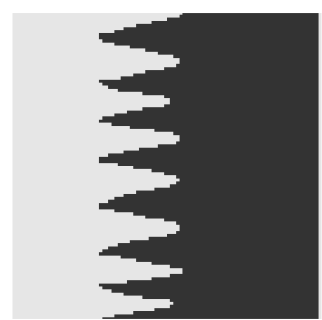

(d) $h_{\text {conv }}^{E W}=400$

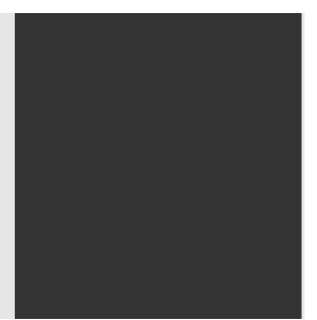

(e) $h_{c o n v}^{E W}=10^{3}$

Fig. 13: Design solutons for the $f_{Q}$ optimization problem

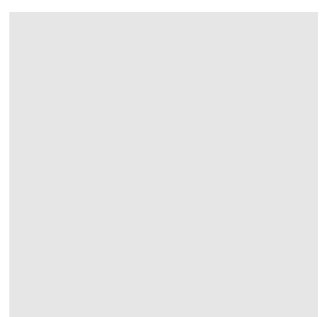

(a) $h_{\text {conv }}^{E W}=3000$

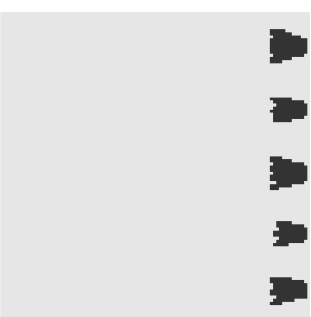

(b) $h_{\text {conv }}^{E W}=5000$

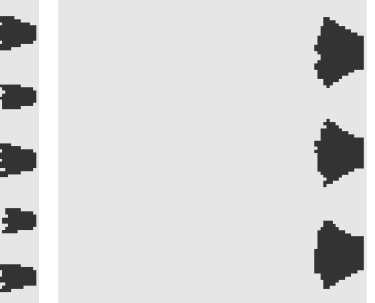

(c) $h_{\text {conv }}^{E W}=8000$

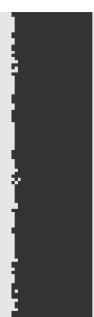

(d) $h_{\text {conv }}^{E W}=20000$

Fig. 14: Optimized designs for the $f_{\mu}$ optimization problem

A design optimized for $f_{P}$ and a design optimized for $f_{\eta}$ have been plotted in Figs. 15a-15b and Figs. 15c-15d, respectively. The design solutions indeed show very different and complex topological designs, and the example demonstrates that the framework actually is capable of optimizing an arbitrary set of boundary conditions.

\subsection{The $p$ - $n$ generator}

The seventh and last numerical example concerns the so-called $p$-n generator (PNG) problem. The problem and the material parameters are inspired by the work of Angst (2016). p- $n$ generators (PNGs) have - compared to conventional configurations of TEGs - an advantage in high temperature applications, as the electrodes in PNGs are disconnected from the heat input surfaces, which may reduce the thermal stress and wear on the electrodes. In conventional TEGs the electrodes are directly connected to the heat input surfaces, please confer Fig. 3, where the electrodes on PNGs are connected to thermally insulated surfaces, please confer Fig. 16. Due to lower temperatures on the insulated surfaces this configuration reduces the thermal stresses in the electrodes during operation. PNGs are prone to relatively poor theoretical performance compared conventional TEGs, however with topology optimization and the framework presented in this study we are able to reduce the performance gap between PNGs and convectional TEGs. The design problem takes basis in the schematic in Fig. 16. The material parameters used in the problem are
Table 6: Boundary conditions for the PNG problem

\begin{tabular}{lcccc}
\hline & \multicolumn{4}{c}{ Boundary } \\
\cline { 2 - 5 } & $\Gamma^{N}$ & $\Gamma^{S}$ & $\Gamma_{E}^{E}$ & $\Gamma_{E}^{W}$ \\
\hline$T_{f l}$ & 1000 & 0 & - & - \\
$h_{\text {conv }}$ & $10^{4}$ & $10^{4}$ & - & - \\
$V$ & - & - & - & 0 \\
$\sigma_{\text {per }}$ & - & - & $10^{10}$ & - \\
$z_{\text {imp }}$ & - & - & $z_{i m p}$ & - \\
\hline
\end{tabular}

academic, however, they are adequate for this specific design problem as Material A and B are equal in magnitude for $\alpha, \sigma$ and $\kappa$, but with opposing operational sign in $\alpha$.

The boundary conditions of the optimization problem has been listed in Tab. 6, and the corresponding design optimized for $f_{P}$ have been plotted in Fig. 17. Several complicated topological design features occur in the optimized design such as asymmetry around $\{x, y\}=\left\{L_{x} / 2, y\right\}$, spike-shaped transitions and isolated islands of different material phases. The unintuitive and complex design features and comparisons with design solutions in Angst (2016), en-light that the proposed topology optimization approach is an effective strategy to optimize such problems. 


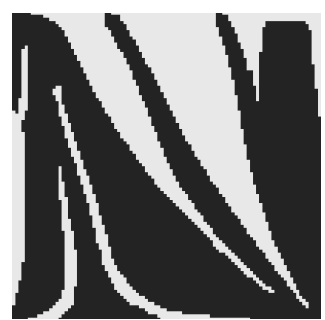

(a) $f_{P}$, set 1

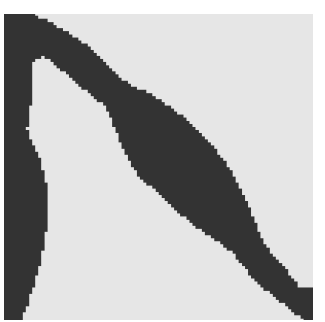

(b) $f_{\eta}$, set 1

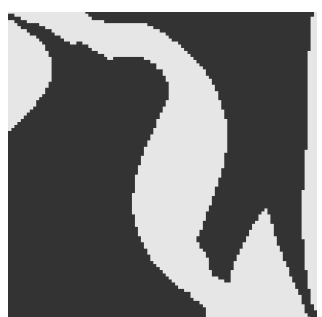

(c) $f_{P}$, set 2

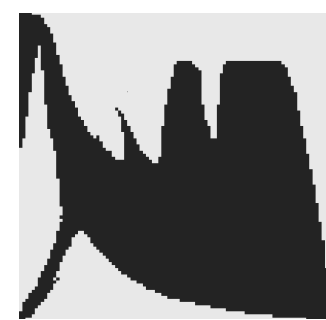

(d) $f_{\eta}$, set 2

Fig. 15: Design soluations for the asymmetric boundary conditions problem

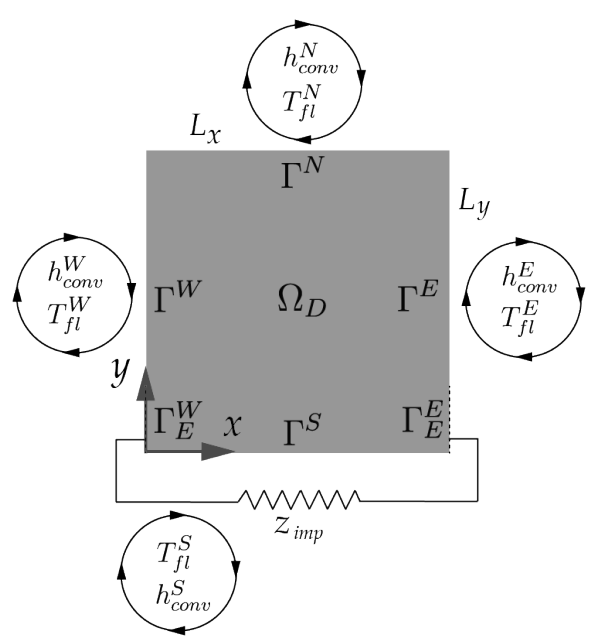

Fig. 16: Schematic of the PNG design problem

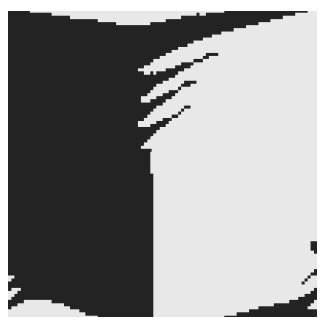

Fig. 17: The design solution for the PNG problem

\section{Discussion and conclusion}

A density-based topology optimization approach for thermoelectric energy conversion problems has been proposed. The versatile framework supports physically realistic convective boundary conditions, temperature dependent material parameters and objective functions relevant to thermoelectric generators and coolers.

The framework is based on a fully coupled non-linear thermoelectric finite element model. The framework distributes two different thermoelectric active materials in a two dimensional design space in order to optimize for some performance measure. The study reveals new insight in physical and topological effects and shows potential performance improvements in the field of thermoelectric energy conversion. The design solutions depend on the boundary conditions, the material parameters and the objective functions. To obtain high performing thermoelectric generators and coolers, it is therefore critical to take the device application into consideration in the design phase.

The design solutions are physically realizable and the framework can easily be applied on physical realistic material parameters and model dimensions. Relevant implementation details with respect to the framework are stated.

The study demonstrates that the proposed approach indeed is well-suited for thermoelectric energy conversion problems. The study may provide guidance for future research in the pursuit at achieving large-scale commercial applications of thermoelectric generators and coolers.

\section{Acknowledgements}

The authors acknowledge the financial support received from the TopTen project sponsored by the Danish Council for Independent Research (DFF-4005-00320).

\section{References}

Sebastian Angst. Complex dynamics and performance of inhomogeneous thermoelectrics. PhD thesis, Von der Fakultt fr Physik der Universitt Duisburg-Essen, 2016.

Elena E Antonova and David C Looman. Finite Elements for Thermoelectric Device Analysis in ANSYS. International Conference on Thermoelectrics, pages 1-4, aug 2000.

Martin Bendsøe and Ole Sigmund. Topology Optimization - Theory, Methods and Applications. Springer, 2003. 
Martin Philip Bendsøe and Noboru Kikuchi. Generating optimal topologies in structural design using a homogenization method. Computer methods in applied mechanics and engineering, 71(2):197-224, 1988.

Daniel Champier. Thermoelectric Generators: A Review of Present and Future Applications, pages 203-212. Springer International Publishing, Cham, 2017.

Robert D. Cook, David S. Malkus, Michael E. Plesha, and Robert J. Witt. Concepts and applications of finite element analysis. John Wiley \& Sons, 4th edition, 2007.

Peter Deuflhard. Newton Methods for Nonlinear Problems. Springer, Nov 2014.

$\mathrm{H}$ Julian Goldsmid. Introduction to thermoelectricity, volume 121. Springer Science \& Business Media, 2009.

Alexander Heghmanns and Michael Beitelschmidt. Parameter optimization of thermoelectric modules using a genetic algorithm. Applied Energy, 155:447-454, 2015 .

David Michael Rowe. Thermoelectrics handbook: macro to nano. CRC press, 2005.

Akihiro Sakai, Tsutomu Kanno, Kouhei Takahashi, Hiromasa Tamaki, Hideo Kusada, Yuka Yamada, and Hiroya Abe. Breaking the trade-off between thermal and electrical conductivities in the thermoelectric material of an artificially tilted multilayer. Scientific reports, 4:6089, 2014.

Ole Sigmund. Systematic design of electrothermomechanical microactuators using topology optimization. Modelling and Simulation of Microsystems, Semiconductors, Sensors and Actuators, pages 1492-1500, 1998.

Ole Sigmund. Manufacturing tolerant topology optimization. Acta Mechanica Sinica/Lixue Xuebao, 25 (2):227-239, 2009.

Ole Sigmund. On the usefulness of non-gradient approaches in topology optimization. Structural and Multidisciplinary Optimization, 43(5):589-596, Mar 2011.

Ole Sigmund and Kurt Maute. Topology optimization approaches. Structural and Multidisciplinary Optimization, 48(6):1031-1055, 2013.

Krister Svanberg. The method of moving asymptotesa new method for structural optimization. International journal for numerical methods in engineering, 24(2): 359-373, 1987.

Takezawa and M Kitamura. Geometrical design of thermoelectric generators based ontopology optimization. International Journal for Numerical Methods in Engineering, 90(April):1885-1891, 2012.

Zhiting Tian, Sangyeop Lee, and Gang Chen. A comprehensive review of heat transfer in thermoelectric materials and devices. Ann. Rev. Heat Transfer, 17:
425-483, 2014

Terry M Tritt and M a Subramanian. Thermoelectric Materials, Phenomena, and Applications : A Bird's Eye View. MRS Bulletin, 31(March):188-198, 2006.

T S Ursell and G J Snyder. Compatibility of segmented thermoelectric generators. Audio, Transactions of the IRE Professional Group on, pages 412-417, August 2002. ISSN 0-7803-7683-8.

Cronin B. Vining. An inconvenient truth about thermoelectrics. Nature Materials, 8(2):83-85, 2009.

Fengwen Wang, Boyan Stefanov Lazarov, and Ole Sigmund. On projection methods, convergence and robust formulations in topology optimization. Structural and Multidisciplinary Optimization, 43(6):767-784, dec 2011.

Osamu Yamashita, Shoichi Tomiyoshi, and Ken Makita. Bismuth telluride compounds with high thermoelectric figures of merit. Journal of Applied Physics, 93 (1):368-374, 2003.

Yang Yang, S H Xie, Feiyue Ma, and Chi Hou Lei. On the effective thermoelectric properties of layered heterogeneous medium. Journal of Applied Physics, 111(1):3510, January 2012.

Yang Yang, Feiyue Ma, Chi Hou Lei, Liu YY, and Jiangyu Li. Is thermoelectric conversion efficiency of a composite bounded by its constituents? Applied Physics Letters, 102(5):53905, January 2013.

SP Yushanov, LT Gritter, JS Crompton, and KC Koppenhoefer. Multiphysics analysis of thermoelectric phenomena. In Seventh Annual Conference on Multiphysics Modeling and Simulation. Proceedings of the 2011 COMSOL Conference, Boston, USA, 2011. 